\title{
Diet and antibiotic growth promoter alter the intestinal microbiota of beef cattle
}

José Antonio Bessegatto[]", Laís Rezende Paulino ${ }^{[a]}$, Bruna Parapinski dos Santos ${ }^{[a]}$, Júlio Augusto Naylor Lisbôa $a^{[a]}$, Amauri Alcindo Alfieri[a], Carlos Henrique Montemor ${ }^{[a]}$, Marcio Costa ${ }^{[b]}$

\footnotetext{
[a] Universidade Estadual de Londrina (UEL), Londrina, PR, Brasil

[b] Université de Montréal (UdeM), Montréal, Québec, Canada
}

*Corresponding author

e-mail: bessegatto.ja@gmail.com

\section{Abstract}

Microbial communities are involved with better weight gain by increasing nutrients harvested from food. The intestinal microbiota can be altered by many factors such as diet, management, stress, diseases, and antibiotics. Antibiotics have been given to farm animals to promote weight gain, but the longitudinal alterations of the intestinal microbiota of beef cattle after confinement into feedlots and after virginiamycin exposure has not been investigated using DNA sequencing. To verify the impact of feedlot diet and virginiamycin used as growth promoter on the intestinal microbial community of beef cattle at different moments, eighteen steers entering a feedlot facility had fecal samples collected at arrival, mid feedlot (26 days) and at exit to slaughtering (100 days). Diet consisted of corn silage (28\%), orange pulp silage (12\%) and concentrate (60\%). One group received virginiamycin (ATB) with predictable ingestion of $0.94 \mathrm{mg} / \mathrm{kg} /$ day and the other was kept without the antibiotic as a control (CON). DNA was extracted and the v4 region of the 16S rRNA gene was sequenced with Illumina MiSeq platform. Body weight was measured at arrival and exit. Parsimony test was used to compare communities between groups and the different moments and a t-test to compare body weight and average daily gain. Entrance into feedlot induced changes in microbiota composition when comparing arrival to mid feedlot $(\mathrm{P}<0.001)$. The bacterial community found in CON was statistically different from the one present in ATB at mid feedlot $(\mathrm{P}<0.001)$. Interestingly, animals receiving virginiamycin had no significant changes in microbial composition from mid to exit $(\mathrm{P}=0.054)$. At exit, no differences were observed between treatments $(P=0.220)$. The major changes after entrance were an increase in Proteobacteria and decrease in Verrucomicrobia and Bacteroidetes $(\mathrm{P}<0.001)$. Bifidobacterium had greater abundance amongst CON animals compared to ATB at mid feedlot $(\mathrm{P}=0.002)$ and exit $(\mathrm{P}<0.001)$. No difference in performance were observed between treatments $(\mathrm{P}>0.05)$, but LefSe analysis revealed two 
genera associated with high daily weight gain, Ruminococcus and Sporobacter, and two with low weight gain, Peptostreptococcaceae incertae sedis and Clostridium. In this study, the similarity of bacterial communities between mid feedlot and exit in animals treated with virginiamycin is suggestive of a faster adaptation of the microbiota. The decrease of Bifidobacterium spp. in treated animals deserves better attention, since some strains of the bacterium have been used in animal production systems (e.g.: probiotics). This data can be used as the basis for the development of new diet adaptation strategies and for alternatives to the use of antibiotics in feedlot cattle. In conclusion, diet is the main factor affecting the intestinal microbial communities of beef cattle and virginiamycin affected mainly rare species of the intestinal bacteria. No benefits on weight gain were observed with the use of virginiamycin as growth promoter. 